\title{
Molecular Packing Effects on the Optical Spectra and Triplet Dynamics in Oligofluorene Films
}

\author{
Juan Cabanillas-Gonzalez, ${ }^{*},{ }^{\dagger}$ Calogero Sciascia, ${ }^{\dagger}$ Guglielmo Lanzani, ${ }^{\dagger}$ Stefano Toffanin,, \\ Raffaella Capelli, $₫$ Miguel C. Ramon, $₫$ Michele Muccini, $₫$ Johannes Gierschner, $₫$ \\ Tsyr-Yuan Hwu," and Ken-Tsung Wong"
}

IFN-CNR, Dipartimento di Fisica, ULTRAS-INFM, Politecnico di Milano, Milano 20133, Italy, Istituto per lo Studio dei Materiali Nanostrutturati (ISMN), Consiglio Nazionale delle Ricerche, via P. Gobetti 101, I-40129 Bologna, Italy, Laboratory for Chemistry of Novel Materials, University of Mons-Hainaut, Place du Parc 20, B-7000 Mons, Belgium, and Madrid Institute for Advanced Studies IMDEA in Nanoscience, UAM, Modulo, C-IX, Av. Tomás y Valiente 7, Campus de Cantoblanco, 28049 Madrid, Spain, and Department of Chemistry, National Taiwan University, Taipei 106, Taiwan

Received: May 19, 2008; Revised Manuscript Received: July 3, 2008

\begin{abstract}
We report on the triplet spectra and dynamics in two types of oligomeric films deposited by two different techniques: thermal evaporation and spin coating. The different molecular arrangement in both films is manifested in a red shift of the absorption, PL, and $\mathrm{T}_{1}-\mathrm{T}_{n}$ absorption spectra of the sublimated film relative to the spin-coated one. Triplet recombination dynamics studied with steady-state photoinduced absorption (PA) spectroscopy follow a dispersive bimolecular recombination model away from the trap filling regime. We obtained values for the triplet bimolecular recombination ratio $(\beta)$ of $3.4 \times 10^{-14}$ and $1.1 \times 10^{-15} \mathrm{~cm}^{3}$ $\mathrm{s}^{-1}$ for evaporated and spin-coated film, respectively, the difference being attributed to diverse molecular arrangement in both films.
\end{abstract}

\section{Introduction}

The efficiency of organic light emitting diodes (OLEDs) has experienced a notable enhancement in the recent years due to the overcoming of some of their main theoretical limitations. ${ }^{1-4}$ One of them is the quantum spin statistics prediction of a population of singlets and triplets from charge recombination in a ratio 1:3. Although some deviations from this prediction were reported in long chain polymers, it appears that triplets are the main product of charge recombination, at least concerning small organic molecules and short oligomers. ${ }^{5-9}$ On account of the long lifetime for triplet radiative recombination due to the dipole forbidden $\mathrm{T}_{1}-\mathrm{S}_{0}$ transition as well as additional nonradiative channels, the efficiency of triplet emission is very low, limiting therefore the device performance. This limitation can be circumvented by including a heavy metal atom in the molecular structure which enhances spin orbit coupling and mixes singlet and triplet states. ${ }^{10}$ Moreover, new strategies consisting of management of singlets and triplets via efficient energy transfer (Förster and Dexter) to highly emissive emitters dispersed in the organic bulk have led to significant enhancement in the performance of OLEDs. ${ }^{11,12}$ At this point, understanding the annihilation processes between species involved in radiative emission, i.e., singlets, triplets, and polarons, becomes crucial for further efficiency improvements. Singlet-singlet and triplet-triplet annihilation are two processes which are strongly dependent on the population density of singlets and triplets due to their corresponding low capture (Förster and Dexter) radius. ${ }^{13}$

\footnotetext{
* Corresponding author. E-mail: juan.cabanillas@polimi.it. TF +39(0)223996055, FAX: +39(0)223996126

+ IFN-CNR

¥ Istituto per lo Studio dei Materiali Nanostrutturati (ISMN).

$\S$ University of Mons-Hainaut and Madrid Institute for Advanced Studies IMDEA in Nanoscience.

"National Taiwan University.
}

These mechanisms may occur as a one-step process if the average separation between the species involved is below the capture radius, or else a multistep process via diffusion between adjacent molecules. Since diffusion is controlled by intermolecular separation of the molecules, film morphology may play an important role on annihilation reaction rates. Evidence for an increase in diffusion upon molecular packing is found on the enhancement in charge photogeneration in $\mathrm{TiO}_{2}$ dye sensitized solar cells based on liquid crystalline porphyrin layers with respect to that of the crystalline phase. ${ }^{14}$ This effect has often been explained in terms of an increase in charge mobility within quasi-one-dimensional porphyrin stacks, rather than enhanced singlet or triplet diffusion to dissociating $\mathrm{TiO}_{2} /$ porphyrin interfaces. However, Savenije et al. demonstrated recently that the triplet diffusion length in a lamellar porphyrin layer increases from 0.95 to $9.6 \mathrm{~nm}$ upon annealing over the crystalline-discotic phase, where parallel organization of molecules leads to short interlamellar separation. ${ }^{15}$

In this work, we address the influence of molecular packing on triplet diffusion in fluorene trimers. We employed two types of oligomers: ter(9,9-diarylfluorene) (T3) and 9,9, $9^{\prime}, 9^{\prime}, 9^{\prime \prime}, 9^{\prime \prime}$ hexa-sec-butyl-[2,2'; $\left.7^{\prime}, 2^{\prime \prime}\right]$ terfluorene (F3), the chemical structures being depicted in Figure 1. Polyfluorenes are materials of technological interest which combine interesting optical properties such as high photoluminescence (PL) quantum efficiency in the solid state ${ }^{16,17}$ and low energy threshold for amplified spontaneous emission (ASE) ${ }^{18,19}$ with notable charge transport properties. ${ }^{20}$ We employ different film deposition techniques (spin coating and thermal evaporation) to induce diverse intermolecular separation in films, reflected in an energetic shift of the absorption, $\mathrm{PL}$, and $\mathrm{T}_{1}-\mathrm{T}_{n}$ triplet photoinduced absorption (PA) spectra. On account of quantum-chemical calculations, we infer that the changes in the optical spectra cannot be ascribed to intramolecular side chain effects but to changes of polarization 


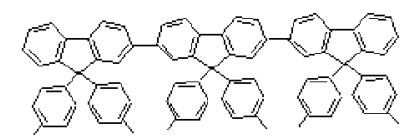

T3

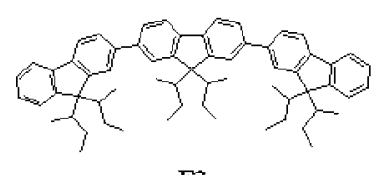

$\mathrm{F} 3$

Figure 1. Chemical structures of $\mathrm{T} 3$ and $\mathrm{F} 3$.

interactions in the films arising from different molecular packings. We monitor with steady-state PA measurements the dynamics of triplet recombination in both oligomeric films, observing different recombination behaviors. These are explained in terms of larger triplet exciton diffusion in the film with higher molecular condensation.

Description of Methods. A. Experimental Methods. F3 films of approximately $100 \mathrm{~nm}$ were prepared by spin coating at $2000 \mathrm{rpm} 20 \mathrm{~g} / \mathrm{L}$ chloroform solutions onto glass substrates. The low solubility of T3 in organic solvents prevented film processing by spin coating. Sublimated T3 films of $93 \mathrm{~nm}$ thickness were instead prepared by thermal evaporation at high vacuum onto silica substrates with a $0.4 \AA / \mathrm{s}$ deposition rate. PA spectroscopy was performed using the $10 \mathrm{~mW}$ output power at $375 \mathrm{~nm}$ of an Omicron diode laser as the excitation source. The laser beam was chopped mechanically at frequencies in the range $30-1500 \mathrm{~Hz}$ and focused in an $\sim 10 \mathrm{~mm}^{2}$ spot on the sample. The probe light from a $30 \mathrm{~W}$ Spectral Products tungsten lamp was spectrally filtered with a monochromator and focused with a set of spherical mirrors on the photoexcited sample area. Phase sensitive detection of the probe transmitted through the sample was provided by a Stanford Research Systems lock-in amplifier and an amplified Si photodiode. The in-phase and outof-phase components provided by the lock-in were recorded with a Labview program which synchronized wavelength scanning and detection. Single wavelength detection measurements were performed at different chopping frequencies and different intensities to study the dynamics of triplet recombination. Measurements were performed at $60 \mathrm{~K}$ by enclosing the sample in an Oxford Instruments closed circuit helium cryostat with temperature controller. Atomic force microscropy (AFM) images were collected using a NT-MDT Solver Scanning Probe Microscope in tapping mode with the samples kept in air. Amplified spontaneous emission (ASE) properties of T3 and F3 thin films were measured at $20 \mathrm{~K}$ by a frequency tripled $355 \mathrm{~nm}$ line of a $10 \mathrm{~Hz}, 25$ pulse duration, Q-switched Quantel Nd:YAG laser being the output signal focused on a fiber-coupled Hamamatsu CCD spectrometer by a lens system.

B. Theoretical Methods. Quantum-chemical calculations of the lowest optical transitions of 9, $9^{\prime}$-substituted oligofluorenes with $n=1-3$ repetition units and $\mathrm{R}=$ methyl $(\mathrm{Me})$ and phenyl $(\mathrm{Ph})$ were performed to elucidate the intramolecular substituent effect on the electronic transition. The molecules were optimized at the DFT level, using the B3LYP functional and the 6-311G* basis set. To investigate the subtle substituent effects, we imposed the highest possible symmetry $\left(C_{2 v}\right.$ for $n=$ odd, $C_{2 h}$ for $n=$ even), in order to not mask the substituent effect by the differences in the electronic structure, as induced by different torsional angles. The electronic structure was calculated at the semiempirical ZINDO/S (intermediate neglect of differential overlap as paramaterized by Zerner et al. $)^{21}$ level coupled to a CIS (configuration interaction singles) scheme, taking into account all $\pi, \pi^{*}$ type orbitals, providing a good compromise

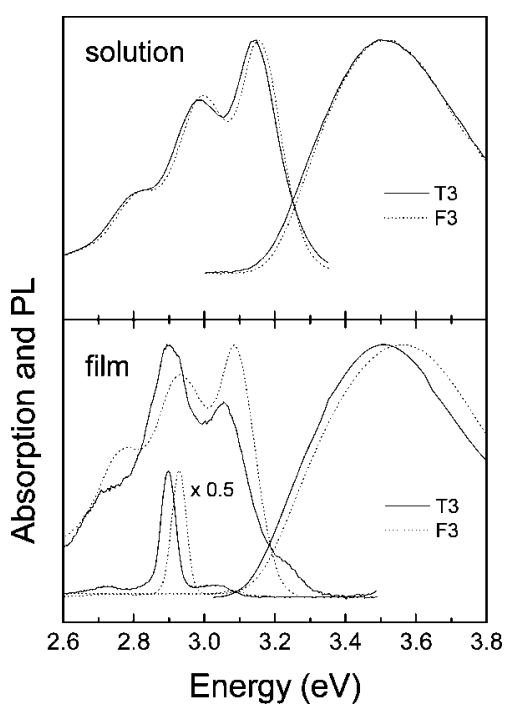

Figure 2. Normalized absorption and PL spectra of T3 and F3 in solution (upper figure) and film together with ASE spectra of both materials (lower figure).

of reliability and efficiency for the calculations of optical transitions for short and long oligomers. ${ }^{22}$ For comparison, TDDFT calculations were performed at the B3LYP/6-311G* level, which commonly give good results on substituent effects, however, systematically underestimating the absolute transition energies of long oligomers. ${ }^{20}$ All (TD-)DFT calculations were carried out within the Gaussian 03 program package. ${ }^{23}$

\section{Results}

The absorption and PL spectra of T3 and F3 in tetrahydrofuran (THF) solutions and films are displayed in the top and bottom parts of Figure 2, respectively. In THF, both oligomers exhibit very similar spectral features consisting of a broad absorption band and vibronically resolved PL spectra typical of polyfluorenes. T3 absorption and PL are slightly red-shifted by approximately $10 \mathrm{meV}$ with respect to $\mathrm{F} 3$, whereas in toluene solution (not shown) the shift amounts to $20 \mathrm{meV} \cdot{ }^{24} \mathrm{In}$ film, the spectra become broader with respect to solution particularly for the case of F3 (Figure 2), whereas the red shift of T3 with respect to F3 increases to $54 \mathrm{meV}$. Upon pulsed excitation with high fluences, both T3 and F3 PL experience a spectral narrowing due to ASE, the peaks being shifted in the same direction by $81 \mathrm{meV}$. We remark however that in this case the differences cannot be attributed exclusively to intermolecular interactions but also to cavity effects. Owing to different thicknesses of T3 and F3 films, amplification of different modes are supported. Figure 4a,b displays AFM scans of the surface of T3 and F3, respectively, showing for both films amorphous morphologies with surface roughness below $1 \mathrm{~nm}$. The PA spectrum of T3 and F3 (Figure 4c) is dominated by a narrow feature typical of poly(9,9-dioctylfluorene) (PFO) although located at lower energies $(\sim 1.4 \mathrm{eV}){ }^{25-27}$ Previous studies with PA spectroscopy assisted by magnetic fields allowed identification of this band to triplet-triplet absorption from the lowest $\mathrm{T}_{1}$ to a generic upper lying $\mathrm{T}_{n}$ state. ${ }^{28}$ In oligomers, a blue shift of the $\mathrm{T}_{1}-\mathrm{T}_{n}$ transition with respect to that of polymers is expected due to shorter conjugation length and less exciton delocalization, as already reported in fluorene trimers ${ }^{29}$ Interestingly, a red shift of $50 \mathrm{meV}$ of $\mathrm{T} 3$ with respect to F3 is detected, in good agreement with absorption and PL spectra. We monitored the triplet recombination dynamics in $\mathrm{T} 3$ and $\mathrm{F} 3$ by performing PA measurements as a function of frequency $(\omega)$ 


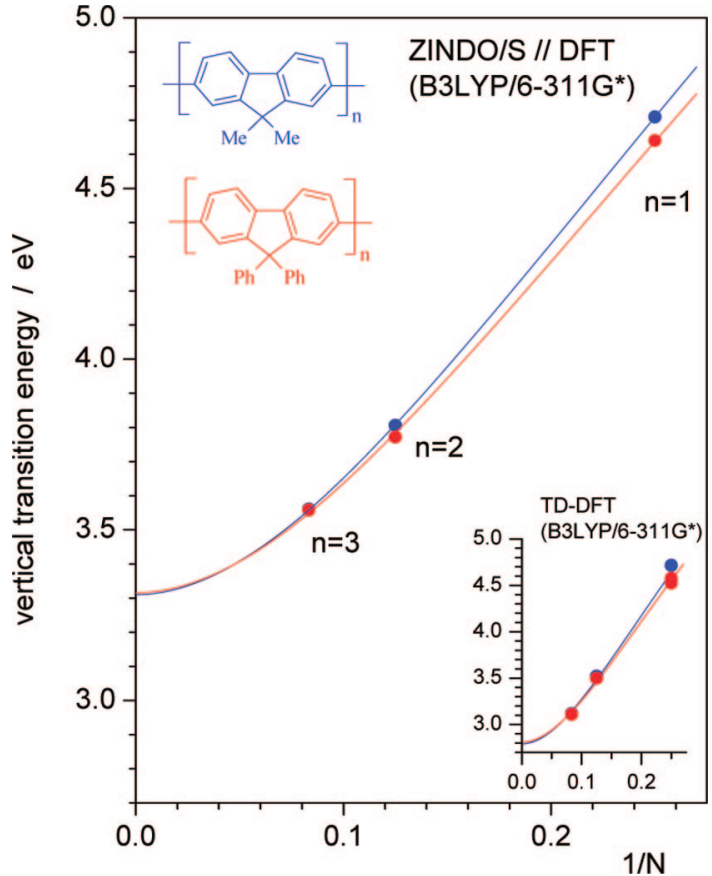

Figure 3. Vertical transitions of 9, $9^{\prime}$-substituted oligofluorenes, as calculated at the ZINDO/S level of theory, based on a DFT (B3LYP/ 6-311G*) geometry optimization. Solid lines are Kuhn fits to the calculated values. Inset: TD-DFT calculations (B3LYP/6-311G*).

and triplet generation rate $(g)$. Note that $g$ is related to the laser fluence $(I)$ through the expression $g=\kappa \gamma I / d$, with $\kappa$ being the ratio of absorbed photons, $\gamma$ the probability of singlets to undergo intersystem crossing to a triplet state ( 0.05 in glassy PFO), ${ }^{25}$ and $d$ the film thickness. The high frequency falloff of the PA signal with $\omega$ (Figure 5) and the weak (strong) sublinear dependence of in (out) of phase PA components with $g$ (Figure 6) is noteworthy. PA values were fitted according to the Cole-Cole relaxation model: ${ }^{30-32}$

$$
\operatorname{PA}(g, \omega) \propto \frac{S\left(g, N_{\mathrm{t}}\right) g \tau_{0}}{1+\left(i \omega \tau_{0}\right)^{\alpha}}
$$

where $0 \leq S \leq 1$ is a function of $g$ and the density of traps $\left(N_{\mathrm{t}}\right) ; \alpha \leq 1$ is a parameter that takes into account dispersive recombination and $\tau_{0}$ is the mean lifetime. In our case, we fit the experimental data with a pure bimolecular recombination model. Extension of eq 1 to bimolecular recombination requires $\tau_{0}\left(\tau_{\mathrm{b}}\right)$ to be given by $g^{-0.5} \beta^{-0.5}$, where $\beta$ holds for the bimolecular recombination rate. The fit parameters obtained are provided in Table 1.

\section{Discussion}

The optical transitions of conjugated chains are strongly affected by their inherent intramolecular electronic properties as well as polarization interactions with the surrounding medium, both contributions determining the shape of the optical spectra. Nevertheless, comparing spectra in the solid state and solution in a nonspecific solvent (such is the case of THF) allows for quantifying each contribution separately. The observed $10 \mathrm{meV}$ red shift of T3 with respect to F3 in THF solution could point to a slight change in the molecular optical properties of oligomers induced by different side chain substitution. Although measurements were perfomed on highly diluted solutions, we cannot completely rule out the presence of aggregates or solute-solvent interactions responsible for the observed dif-

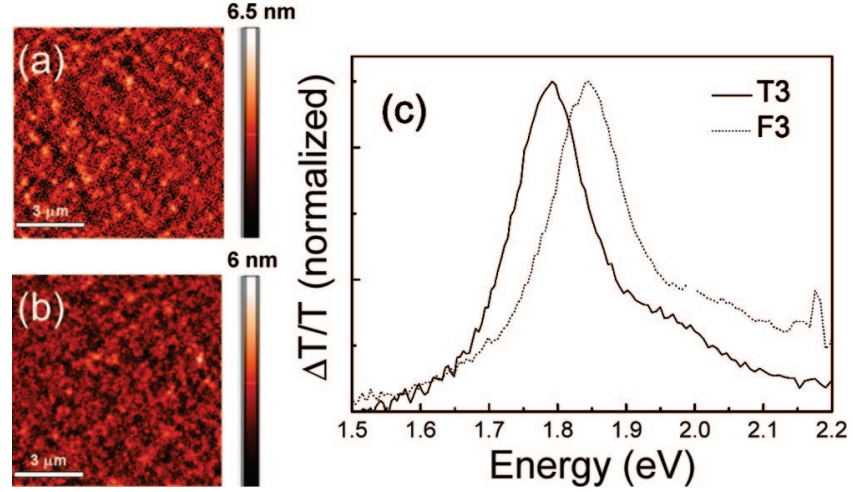

Figure 4. $10 \mu \mathrm{m} \times 10 \mu \mathrm{m}$ tapping mode AFM images of (a) T3 and (b) F3. (c) PA spectrum of T3 at $100 \mathrm{~K}$ and $\omega=80 \mathrm{~Hz}$ modulation frequency.

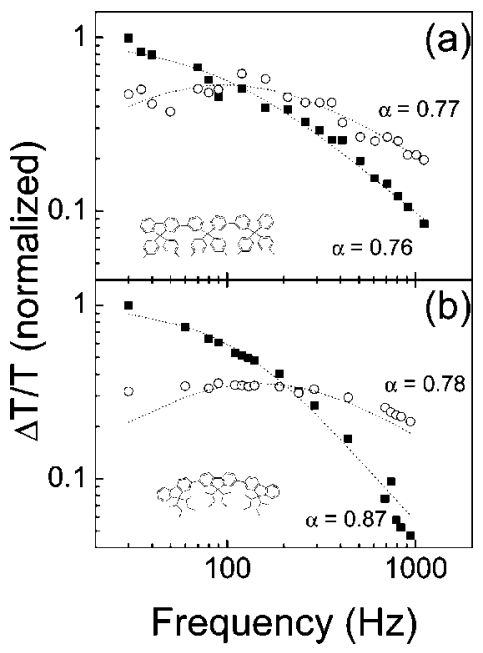

Figure 5. Frequency-dependent PA of (a) T3 and (b) F3. In-phase and out-of-phase components are displayed as filled squares and empty circles, respectively. Curve fits are shown as dotted curves.

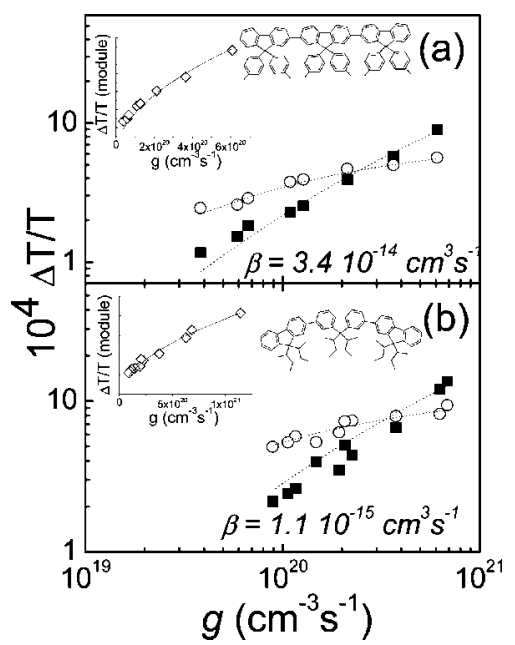

Figure 6. Intensity-dependent PA of (a) T3 and (b) F3. In-phase and out-of-phase components are displayed as filled squares and empty circles, respectively. The different dependence of the in-phase and outof-phase PA points toward a bimolecular triplet recombination process. Curve fits are shown as dotted lines. PA module values are displayed in the insets with their corresponding fit (solid line).

ferences in optical spectra. In order to elucidate a possible influence of chain substituents on the optical spectra, quantumchemical calculations were carried out on oligomers with one, two, and three repetition units. A slight red shift of the lowest 
TABLE 1: Values Obtained from Fit Curves (Note That $\boldsymbol{\tau}_{\mathrm{b}}$ Depends on $g$ Expressed in $\mathrm{cm}^{3} \mathrm{~s}^{-1}$ and $S=1$, Which Implies a Regime Far from Trap Limited Saturation)

\begin{tabular}{ccccc}
\hline & $\alpha$ & $\beta\left(\mathrm{cm}^{3} \mathrm{~s}^{-1}\right)$ & $\tau_{\mathrm{b}}(\mathrm{s})$ & $S$ \\
\hline T3 & $0.76-0.77$ & $3.4 \times 10^{-14}$ & $5.4 \times 10^{6} g^{-0.5}$ & 1 \\
F3 & $0.78-0.87$ & $1.1 \times 10^{-15}$ & $3.0 \times 10^{7} g^{-0.5}$ & 1
\end{tabular}

optical transition is observed for phenyl substituted oligomers, which decreases with oligomer length (Figure 3), essentially vanishing for $n=3$ ( $\Delta E=5 \mathrm{meV}$ at the TD-DFT level). This result confirms the small influence of the side chains in the intramolecular optical properties of $\mathrm{T} 3$ and $\mathrm{F} 3$ and suggests that the $54 \mathrm{meV}$ red shift between $\mathrm{T} 3$ and F3 in the solid state must be mainly attributed to the presence of strong polarization interactions. These effects are particularly notorious in the solid state owing to the larger polarizabilities of $\pi$ conjugated chains compared to solvent molecules. The extent of spectral shift is usually moderate for disordered solids and will depend mainly on the average intermolecular separation, which may be affected by the length and geometry of the chain substituents (side chain dilution). If the solids exhibit a certain degree of order, polarization interactions will be notably enhanced due to chain alignment along preferential directions, leading to a considerably larger energy shift. ${ }^{33}$ Concerning F3 and T3, much evidence provided in previous reports confirms their rather amorphous nature. Differential scanning calorimmetry measurements performed in T3 and F3 revealed marked glass transitions at 200 and $114{ }^{\circ} \mathrm{C}$, respectively. ${ }^{16,34}$ The absence of long-range crystallinity is apparent even in sublimated films, as suggested by featureless X-ray diffraction patterns reported in T3 films. ${ }^{35,36}$ Nevertheless, the existence of small crystalline domains embedded in large disordered regions cannot be completely ruled out. AFM scans on T3 and F3 surfaces show morphologies with the presence of isolated micron scale grains. Taking into account solution and film spectra for each material, T3 displays the larger solid state shift (50 meV for T3 against $10 \mathrm{meV}$ for F3) which could point to a certain degree of order in the former. We should remark however that these energy values remain well below the numbers typically observed in highly ordered systems, e.g., $200 \mathrm{meV}$ for regioregular polyhexylthiophene, ${ }^{20}$ which organizes in parallel one-dimensional stacks. Therefore, it seems reasonable to assign the bathochromic solid state red shift of T3 against F3 to shorter average intermolecular separation in T3, with the possible presence of short-range order associated. A qualitative picture of the effects of molecular density on the optical spectra is provided by the Onsager's spherical cavity model, which relates the gas-to-crystal shift $(\Delta E)$ to the refractive index $(n)$ of the dielectric medium by ${ }^{37}$

$$
\Delta E \propto \frac{f_{\mathrm{vac}}}{R_{0}^{3} E_{\mathrm{vac}}} \frac{n^{2}-1}{2 n^{2}+1}
$$

where $f_{\mathrm{vac}}$ and $E_{\mathrm{vac}}$ are the oscillator strength and energy of the electronic transition in vacuum and $R_{0}$ is the radius of an arbitrary cavity taken to be the same for T3 and F3. Interestingly, the calculated oscillator strength of $\mathrm{F} 3\left(f_{\mathrm{vac}}=2.7\right)$ is somewhat higher than $\mathrm{T} 3\left(f_{\mathrm{vac}}=2.5\right)$ so that $n$, which gives rise to the spectral shift, should be significantly higher in T3. In the absence of anisotropy, large values of $n$ are related to a higher density of T3 molecules ${ }^{38}$ via their relation through the molar refraction. The increase of density in T3, i.e., the reduction of intermolecular separation, might be due to a better interdigitation of the side groups in $\mathrm{T} 3$ compared to $\mathrm{F} 3$. The different procedures employed for film deposition, i.e., thermal evaporation and spin coating, could have a certain influence in molecular packing of T3 and F3 films. Thermal evaporation could allow for a larger molecular reorganization with respect to that of spin cast films, in arrangements where effects of steric side chain repulsion are minimized, leading thereby to a larger molecular density in $\mathrm{T} 3$ with respect to F3 film.

Hereafter, we discuss triplet recombination in both oligofluorenes. It is well accepted that triplet photogeneration in polyfluorene occurs by intersystem crossing from the singlet to the triplet manifold, a process that takes place in approximately $10 \mathrm{~ns} .{ }^{39}$ Subsequently, triplets decay mainly via non-radiative channels, although a small percentage may recombine radiatively to $S_{0}$ by spin flip of one of the two electrons accommodated in the triplet state, emitting phosphorescence. On account of phosphorescence lifetime in polyfluorene and ladder type poly phenylene at $77 \mathrm{~K}$ exceeding $100 \mathrm{~ms},{ }^{40,41}$ triplet radiative recombination can be neglected in the time scale of our measurements $(1-100 \mathrm{~ms})$. Thus, the decrease of PA with the modulation frequency is more likely to be influenced by nonradiative recombination processes. We focus now our attention on the observed PA frequency dependence in T3 and F3. Equation 1 provides good fitting to the data with $\alpha$ values between 0.7 and 0.9 pointing to dispersive recombination dynamics motivated by the presence of non-radiative triplet decay channels. ${ }^{42}$ Energy disorder fuels triplet diffusion to lower energy sites ${ }^{43}$ and opens up new recombination channels such as triplet-triplet annihilation or self-trapping and dissociation at defects. ${ }^{44}$ From fits to the $\omega$-dependent PA, we obtained average triplet decay lifetimes of 1.3 and $13.2 \mathrm{~ms}$ for T3 and F3, respectively. Additional information on the nature of triplet recombination is provided by intensity-dependent PA. It is noteworthy to emphasize three main characteristics of the dynamics. First, the in-phase and out-of-phase components in both oligofluorenes show weak and strong sublinear dependence, respectively. This observation is an unambiguous proof for preferential bimolecular recombination, since according to eq 1 monomolecular recombination would imply the same $g$ dependence for both in-phase and out-of-phase PA components. Full bimolecular recombination has already been reported in PA studies of regiorandom P3HT and F8BT. ${ }^{45,46}$ Second, reasonable agreement was found between the experimental data and curve fits when taking $S=1$, i.e., a regime away from trap filling. This result is interpreted as being due to the low laser fluence employed in this work which does not suffice trap saturation. Taking these considerations into account, it seems reasonable to attribute the bimolecular recombination dynamics to triplet-triplet annihilation involving mobile triplets. Moreover, we note that the bimolecular recombination rate $(\beta)$ of T3 is approximately 30 times larger than that in F3. These differences could be explained as being due to the dependence of triplet diffusion on the intermolecular separation and the different molecular distribution in T3 and F3 films. Triplet diffusion is governed by the Dexter mechanism with a rate of $k \propto e^{-2 r}$, where $r$ is the nearest neighbor molecule. Since Dexter transfer is a short-range interaction which requires wave function overlap between the donor and acceptor, it is therefore highly sensible to intermolecular separations or molecular packing. Accordingly, the larger triplet-triplet annihilation rate in T3 could be attributed to enhanced triplet diffusion due to shorter intermolecular separation with respect to F3 film.

\section{Conclusions}

We studied triplet recombination dynamics, which are relevant for LED applications, in oligofluorene films with 
different molecular packing. The triplet dynamics in both films follow a pure bimolecular recombination model ascribed to triplet-triplet annihilation, observing a 30 times larger annihilation coefficient in $\mathrm{T} 3$ than in F3. We explain these differences as being due to triplet diffusion mediated by the Dexter mechanism, which is expected to be highly sensitive to the intermolecular separation. This work demonstrates that even small changes in molecular packing, regarding density and interaction, lead to large modification in triplet dynamics.

Acknowledgment. J.C.-G., C.S., and G.L. are thankful to Fondazione Cariplo for funding through the TOLEDO project. J.G. is a Ramón y Cajal research fellow, funded by the Spanish Ministry of Science and Education. M.M. acknowledges funding through the BIMORE (N EU-FP6-Marie Curie-035859) and OLAS (EU-FP6-IST-FET -015034) projects and Italian MIUR funding through FIRB RBNE033KMA and FIRB RBIP0642YL NODIS projects.

\section{References and Notes}

(1) Kim, C.; Burrows, P. E.; Forrest, S. R. Science 2000, $288,831$. 750 .

(2) Baldo, M. A.; Thompson, M. E.; Forrest, S. R. Nature 2000, 403,

(3) Forrest, S. R. Nature 2004, 428, 911.

(4) Adachi, C.; Baldo, M. A.; Thompson, M. E.; Forrest, S. R. J. Appl. Phys 2001, 90, 5048.

(5) Wohlgenannt, M.; Tandon, K.; Mazumdar, S.; Ramasesha, S.; Vardeny, Z. V. Nature 2001, 409, 494.

(6) Wohlgenannt, M.; Jiang, X. M.; Vardeny, Z. V.; Janssen, R. A. J. Phys. Rev. Lett. 2002, 88, 197401.

(7) Wilson, J. S.; Dhoot, A. S.; Seeley, A. J. A. B.; Khan, M. S.; Köhler, A.; Friend, R. H. Nature 2001, 413, 828.

(8) Virgili, T.; Cerullo, G.; Lüer, L.; Lanzani, G.; Gadermaier, C.; Bradley, D. D. C. Phys. Rev. Lett. 2003, 90, 246402.

(9) Shuai, Z.; Beljonne, D.; Silbey, R. J.; Bredas, J. L. Phys. Rev. Lett. 2000, 84, 131 .

(10) Lane, P. A.; Palilis, L. C.; O'Brien, D. F.; Giebeler, C.; Cadby, A. J.; Lidzey, D. G.; Campbell, A. J.; Blau, W.; Bradley, D. D. C. Phys. Rev. B 2001, 63, 235206.

(11) Kawamura, Y.; Goushi, K.; Brooks, J.; Brown, J. J. Appl. Phys. Lett. 2005, 86, 071104.

(12) Sun, Y.; Giebink, N. C.; Kanno, H.; Ma, B.; Thompson, M. E.; Forrest, S. R. Nature 2006, 440, 908

(13) Cabanillas-Gonzalez, J.; Fox, A. M.; Hill, J.; Bradley, D. D. C. Chem. Mater. 2004, 16, 4705.

(14) Liu, C. Y.; Tang, H. J.; Bard, A. J. J. Phys. Chem. 1996, 100, 3587.

(15) Kroeze, J. E.; Koehorst, R. B. M.; Savenije, T. J. Adv. Funct. Mater. 2004, 14, 992.

(16) Oyamada, T.; Chang, C.-H.; Chao, T.-C.; Fang, F.-C.; Wu, C.-C.; Wong, K.-T.; Sasabe, H.; Adachi, C. J. Phys. Chem. C 2007, 111, 108.

(17) Cabanillas-Gonzalez, J.; Virgili, T.; Lanzani, G.; Yeates, S.; Ariu, M.; Nelson, J.; Bradley, D. D. C. Phys. Rev. B 2005, 71, 014211.

(18) Lin, H.-W.; Lin, C.-L.; Wu, C.-C.; Chao, T.-C.; Wong, K.-T. Appl. Phys. Lett. 2005, 87, 071910.
(19) Clark, J.; Bazzana, L.; Bradley, D. D. C.; Cabanillas - Gonzalez, J.; Lanzani, G.; Lidzey, D. G.; Morgado, J,.; Nocivelli, A.; Tsoi, W. C.; Virgili, T.; Xia, R. J. Nanophotonics 2008, 2 (1), 023504.

(20) Wu, C.-C.; Liu, T.-L.; Hung, W.-Y.; Lin, Y.-T.; Wong, K.-T.; Chen, R.-T.; Chen, Y.-M.; Chien, Y. Y. J. Am. Chem. Soc. 2003, 125, 3710.

(21) Zerner, M. C. In Reviews in Computational Chemistry; Lipkowitz, K. W., Boyd, D. B., Eds.; VCH: New York, 1994; Vol. 2, p 313.

(22) Gierschner, J.; Cornil, J.; Egelhaaf, H.-J. Adv. Mater. 2007, 19, 173.

(23) Frisch, M. J. Gaussian 03, revision C.02; Gaussian, Inc.: Wallingford, CT, 2004.

(24) The $20 \mathrm{meV}$ shift in toluene is ascribed to specific solute-solvent interactions, which are expected to be more pronounced in $\mathrm{T} 3$, due to the phenyl substituents.

(25) Cadby, A. J.; Lane, P. A.; Mellor, H.; Martin, S. J.; Grell, M.; Giebeler, C.; Bradley, D. D. C.; Wohlgenannt, M. ; An, C.; Vardeny, Z. V. Phys. Rev. B 2000, 62, 15604.

(26) Hayer, A.; Khan, A. L. T.; Friend, R. H.; Köhler, A. Phys. Rev. B 2005, 71, 241302.

(27) Liao, H. H.; Meng, H. F.; Horng, S. F.; Shy, J. T.; Chen, K.; Hsu, C. S. Phys. Rev. B 2005, 72, 113203.

(28) Cadby, A. J.; Lane, P. A.; Martin, S.; Bradley, D. D. C.; Wohlgenannt, M.; An, C.; Vardeny, Z. V. Synth. Met. 2000, 111, 515.

(29) Cabanillas-Gonzalez, J.; Antognazza, M. R.; Virgili, T.; Lanzani, G.; Gadermaier, C.; Sonntag, M.; Strohriegl, P. Phys. Rev. B 2005, 71, 155207. (30) Ngai, K. L.; White, C. T. Phys. Rev. B 1979, 20, 2475.

(31) Epshtein, O.; Eichen, Y.; Ehrenfreund, E.; Wohlgenannt, M.; Vardeny, Z. V. Phys. Rev. Lett. 2003, 90, 046804.

(32) Epshtein, O.; Nakhmanovic, G.; Eichen, Y.; Ehrenfreund, E. Phys. Rev. B 2001, 63, 125206.

(33) Gierschner, J.; Ehni, M.; Egelhaaf, H.-J.; ,Milián Medina, B.; Beljonne, D.; Benmansour, H.; Bazan, G. C. J. Chem. Phys. 2005, 123, 144914.

(34) Sonntag, M.; Strohriegl, P. Chem. Mater. 2004, 16, 4736.

(35) Lin, H. W.; Lin, C. L.; Chang, H. H.; Lin, Y. T.; Wu, C. C.; Chen, Y. M.; Chen, R. T.; Chien, Y. Y.; Wong, K. T. J. Appl. Phys. 2004, 95, 881.

(36) Wong, K. T.; Chien, Y. Y.; Chen, R. T.; Wang, C. F.; Lin, Y. T.; Chiang, H. H.; Hsieh, P. Y.; Wu, C. C.; Chou, C. H.; Su, Y. O.; Lee, G. H.; Peng, S. M. J. Am. Chem. Soc. 2002, 124, 11576.

(37) Onsager, L. J. Am. Chem. Soc. 1936, 58, 1486.

(38) Gierschner, J.; Egelhaaf, H.-J.; Mack, H.-G.; Schweizer, S.; Doser, B.; Oelkrug, D. Synth. Met. 2003, 138, 311-315.

(39) Burrows, H. D.; De Melo, J. S.; Serpa, C.; Arnaut, L. G.; Monkman, A. P.; Hamblett, I.; Navaratnam, S. J. Chem. Phys. 2001, 115, 9601.

(40) Hertel, D.; Setayesh, S.; Nothofer, H. G.; Scherf, U.; Mullen, K.; Bässler, H. Adv. Mater. 2001, 13, 65.

(41) Vu, Y.; Gerhard, A.; Schweitzer, B.; Scherf, U.; Personov, R. I.; Bässler, H. Phys. Rev. Lett. 2000, 84, 1027.

(42) Wohlgennant, M. In Photophysics of molecular materials; Lanzani,

G., Ed.; Wiley-VCH: Berlin, 2005.

(43) Richert, R.; Bässler, H. J. Chem. Phys. 1986, 84, 3567.

(44) Singlet-triplet recombination is excluded owing to short singlet lifetime.

(45) Westerling, M.; Vijila, C.; Osterbacka, R.; Stubb, H. Chem. Phys. 2003, 286, 315 .

(46) Westerling, M.; Vijila, C.; Osterbacka, R.; Stubb, H. Phys. Rev. B 2003, 67, 195201.

JP8044143 